\title{
Lipase-catalyzed acidolysis of palm olein and caprylic acid in a continuous bench-scale packed bed bioreactor
}

\begin{abstract}
Enzymatic acidolysis of refined, bleached and deodorized (RBD) palm olein with caprylic acid was carried out in a continuous packed bed bioreactor to produce structured lipid (SL) that can confer metabolic benefits when consumed. Lipozyme ${ }^{\circledR}$ IM 60 from Rhizomucor miehei, a 1,3-specific lipase, was used as the biocatalyst in this study. After $24 \mathrm{~h}$ of reaction, $30.5 \%$ of the total fatty acid content of the modified oil was found to be caprylic acid, indicating its incorporation into the palm olein. The triacylglycerols (TAGs) of palm olein after acidolysis were separated and were characterized by seven clusters of TAG species with equivalent carbon number (ECN), C28, C30, C32, C34, C36, C38 and C40. Caprylic-oleiccaprylic TAGs were predicted in cluster $\mathrm{C} 32$, which recorded the highest amount, with $35.3 \%$ of the total TAG. Fatty acid composition at the sn-2 position was determined, by pancreatic lipolysis, as C8:0, 9.2\%; C12:0, 2.3\%; C14:0, 1.8\%; C16:0, 21.3\%; C18:0, 4.7\%; C18:1, $60.7 \%$. Iodine value (IV), slip melting point (SMP) and differential scanning calorimetric (DSC) analyses of SL were also performed. In IV analysis, SL recorded a drop of value from 60.4 to 48.2 while SMP was reduced from 13 to $4.2{ }^{\circ} \mathrm{C}$, in comparison to RBD palm olein. DSC analysis of SL gave a melting profile with two low melting peaks of -15.97 and $-11.78^{\circ} \mathrm{C}$ and onset temperatures of -18.43 and $-14.03{ }^{\circ} \mathrm{C}$, respectively.
\end{abstract}

Keyword: Acidolysis, Caprylic acid, RBD palm olein, Enzymatic synthesis, Lipozyme® IM 60, Packed bed bioreactor, Structured lipids 